\title{
Imaging findings of atypical leiomyoma of the urinary bladder simulat- ing bladder cancer: a case report and literature review
}

\section{Size Wu}

Department of Medical Imaging, Affiliated Hospital of Hainan Medical College, Haikou, China

\begin{abstract}
Atypical bladder leiomyoma is a rare bladder tumor that is difficult to be correctly identified by imaging techniques or cystoscopy. We present the imaging characteristics of an atypical bladder leiomyoma and review the relative literature, with the aim of enhancing awareness of the differential diagnosis of bladder leiomyoma, to avoid and reduce misdiagnosis. The imaging characteristics of the atypical leiomyoma were the cauliflower shaped, abundant vascularity, and calcification foci on the surface of the tumor. The patient was misdiagnosed with bladder cancer after an imaging study. The histopathological study established the definitive diagnosis.
\end{abstract}

Keywords: urinary bladder, leiomyoma, ultrasound, computed tomography, magnetic resonance imaging

\section{Introduction}

Benign tumors of the bladder are rare [1,2], and their sonographic features are usually homogenous hypoechoic, with circumscribed margin and few blood vessels on color Doppler ultrasound. The ultrasonographic and contrast enhanced computed tomography characteristics of the typical leiomyoma of the bladder are exemplified in figure 1 and figure 2. However, the bladder tumors may have variant aspects on imaging evaluation, which may cause misinterpretation and be confused with bladder cancer. Imaging description of atypical leiomyoma of the urinary bladder is rare. The aim of this case report is to delineate imaging aspects of an atypical leiomyoma of the urinary bladder.

\section{Case report}

A 49-year-old man presented with gross hematuria, dysuria, and pollakiuria. He complained of a nearly 3-month history of intermittent gross hematuria. Previ-

Received 13.01.2013 Accepted 18.03.2013

Med Ultrason

2013, Vol. 15, No 2, 161-163

Corresponding author: Size Wu MD

Department of Medical Imaging

Affiliated Hospital of Hainan Medical College,

31, Longhua Road, Haikou 570102, China

Tel: 86-0898-66508833; 86-13976609631

Email:wsz074@yahoo.com.cn

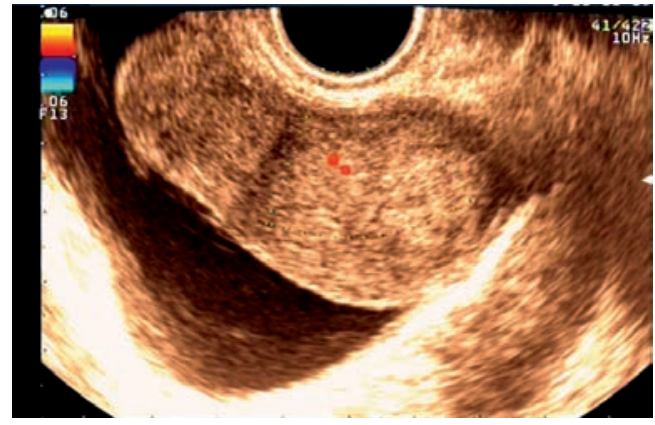

Fig 1. Color Doppler ultrasound reveals a ellipsoid shaped homogeneous hypo $\neg$ echoic mass $63 \mathrm{~mm} \times 36 \mathrm{~mm} \times 31 \mathrm{~mm}$ in the posterior wall of the bladder with circumscribed margin and a few blood vessels

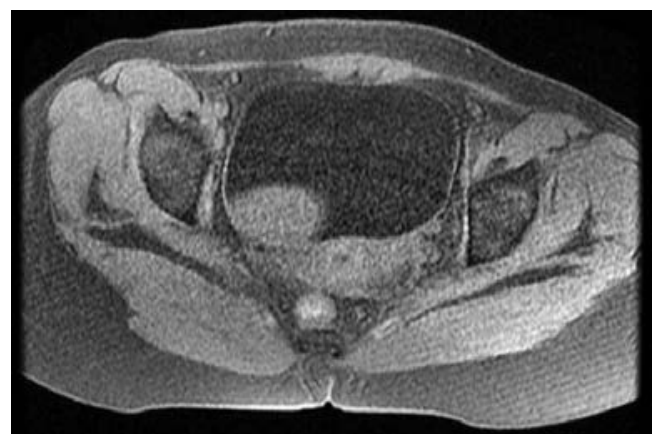

Fig 2. Axial contrast enhanced computed tomography shows moderate homogeneous enhancement of the mass. 


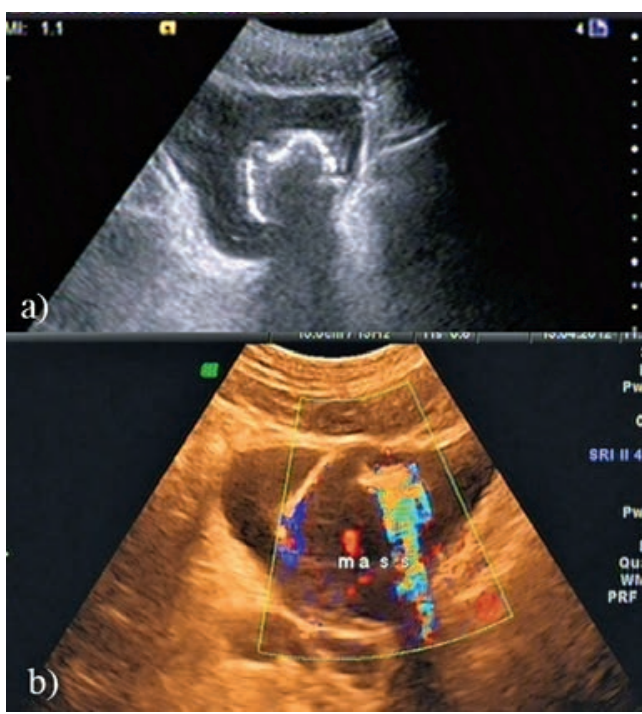

Fig 3. a) Color Doppler ultrasound reveals a cauliflower like shaped heterogenous hypo $\neg$ echoic mass in the neck of the bladder with scallop hyper $\frown$ echoic surface and marked posterior acoustic attenuation; b) Color Doppler flow imaging shows the surface of the mass presenting distinct twinkling and several blood vessels in the mass.

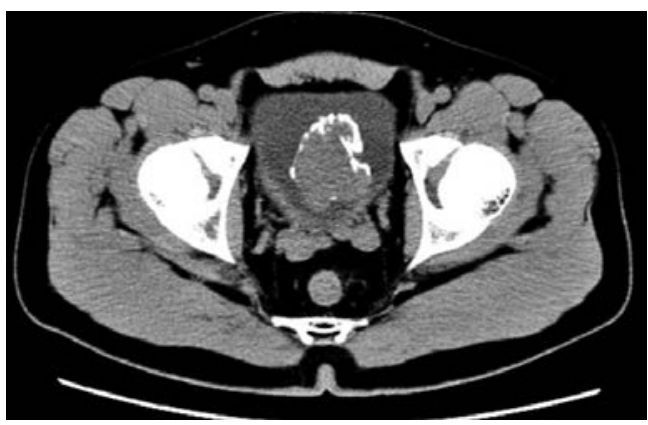

Fig 4. Axial contrast enhanced computed tomography shows a cauliflower like appearance mass, $46 \mathrm{~mm} \times 42$ $\mathrm{mm} \times 40 \mathrm{~mm}$, arising from the left side of the bladder neck with homogeneous centripetal enhancement.

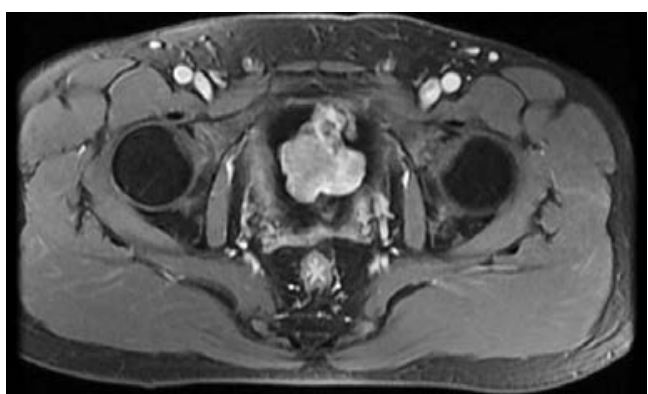

Fig 5. Axial T1 weighted contrast enhanced magnetic resonance imaging shows an irregular shaped mass with cauliflower like appearance arising from the left side of the bladder neck, presenting enhancement of heterogeneous hyperintense. ous ultrasound examination of the bladder was suggestive of bladder stone and cystitis and he was treated for diagnosis. He denied any other medical problems. Physical examination did not reveal any particular findings. Microscopic examination of the urine showed few white blood cells and abundant red blood cells. Blood urea and serum creatinine were within normal limits. The other results of laboratory evaluation were also within the normal limits. Transabdominal ultrasound (US), contrast enhanced computed tomography $(\mathrm{CT})$, magnetic resonance imaging (MRI), and cystoscopy were performed.

US, including Doppler study revealed an irregular shaped heterogenous hypoechoic mass in the neck of the bladder, with cauliflower-like hyperechoic surface, marked posterior acoustic attenuation, a distinct twinkling sign, and abundant blood vessels (fig 3), with resistive index of 0.75 in one of the arterial vessel. The rest of the bladder's walls, the kidneys, the ureters, and the prostate showed no abnormality at US. Intravenous urography revealed a large filling defect within the bladder, and no abnormal findings of kidneys and ureters. Unenhanced CT showed an irregular shaped mass measuring $50 \mathrm{~mm} \times 46 \mathrm{~mm} \times 43 \mathrm{~mm}$ in size in the bladder, with cauliflower like appearance, and density $39 \mathrm{HU}$ except the scallop-like higher dense rim; on contrast enhanced CT, the mass showed homogeneous centripetal enhancement, and enhancement of the mass was $57 \mathrm{HU}$ (fig 4). The mass arises from the left side of the bladder neck and separation from the prostate was obscured. MRI revealed an irregular shaped mass arising from the left side of the bladder neck, presented isointense signal on T1 and T2 weighted images, and the surface was uneven, with cauliflower like appearance, the focal wall of the bladder was infringed thoroughly (fig 5 ). The tumor was homogenously enhanced after injection of gadolinium.

Cystoscopy revealed a cauliflower like tumor at the bladder neck with ulcers and calcification foci or uratosis on the coarse surface. Biopsy of the mass disclosed a submucosal neoplasm composed of fascicles of smooth muscle proliferation suggesting a leiomyoma. Histopathological examination showed the proliferation of spindle-shaped cells with eosinophilic cytoplasm and muscular and fibrous tissue with fibrous stroma. The nuclei of the cells were cigar-shaped and centrally located. No evidence of mitotic figures, coagulative T-cell necrosis or atypia was seen. Immunohistochemistry showed a positive expression for smooth muscle action and a $10 \%$ expression for Ki-67. These findings were consistent with a diagnosis of benign leiomyoma. The final diagnosis was leiomyoma of the bladder without a malignant component and the tumor was resected transurethrally. 


\section{Discussion}

Leiomyoma is a rare benign tumor of the urinary bladder, accounting for $0.43 \%$ of bladder tumors according to a study based on data from three urological centers in Spain [3]. It may occur at different ages [3-5], can be asymptomatic, or can present in a varied manner, depending on the location of the tumor, with obstructive symptoms, irritating symptoms, hematuria, pelvic mass, and pelvic and flank pain [3-8]. Leiomyomas arise in the submucosa, but the growth may be submucosal (7\%), intravesical (63\%), or extravesical (30\%) [5].

Patients with leiomyoma of the bladder are often found at imaging after referring to clinicians for urinary frequency, urinary urgency, gross hematuria, and other symptoms; but some are asymptomatic [3-5]. It exhibits characteristics similar to those of uterine myomas on US, CT and MRI [3-8]. US, CT, MRI, and cystoscopy are valuable diagnostic tools that can depict the morphology and anatomic location of leiomyomas [3-11].

Usually US is the initial imaging modality of choice [3]; on color Doppler flow imaging, blood vessel of leiomyoma can be depicted [7]. In our case, the irregular shaped heterogenous hypoechoic mass with cauliflower like hyperechoic surface and evident posterior acoustic attenuation together with the abundant blood vessels and higher RI made the lesion mimic a bladder cancer. The presence of twinkling sign, hyperechoic rim and marked posterior acoustic attenuation revealed there were stone or calcification foci [9], which led to the misdiagnosis as a bladder stone at first US examination. Although US has a good diagnostic accuracy for focal nodular lesions of the bladder wall, its capability of characterizing focal bladder wall abnormalities is poor [12-14]. The result of this case report supports the study by Horstman et al [14].

CT and MRI are useful for the evaluation of bladder tumors. On CT, typical leiomyoma of the bladder usually presents as a round like hypodense mass with circumscribed margin; on contrast enhanced CT, it shows centripetal homogeneous enhancement $[3,4,6]$. On MRI, typical leiomyomas are usually visualized as low-intensity masses both on $\mathrm{T} 1$ and $\mathrm{T} 2$ weighted sequences with a smooth surface, while degenerative leiomyomas have a heterogenous signal intensity; on contrast enhanced MRI (gadolinium), some leiomyomas show homogeneous enhancement, while other are not $[10,11]$. In our case, $\mathrm{CT}$ and MRI of the leiomyoma demonstrated irregular cauliflower shaped and homogenous enhancement, the relationship of the tumor to the bladder neck and prostate was delineated clearly. These are different from common bladder leiomyoma described in previous published cases, and thus led to misdiagnosis.

\section{Conclusions}

Atypical leiomyoma of the bladder has manifestations simulating bladder cancer, and is difficult to be identified on US, CT, MRI, and cystoscopy. A definitive diagnosis of the bladder lesion depends on biopsy and histopathological study.

\section{References}

1. Cowan NC, Crew JP. Imaging bladder cancer. Curr Opin Urol 2010; 20: 409-413.

2. Wong-You-Cheong JJ, Woodward PJ, Manning MA, Sesterhenn IA. From the Archives of the AFIP: neoplasms of the urinary bladder: radiologic-pathologic correlation. Radiographics 2006; 26: 553-580.

3. Blasco Casares FJ, Sacristán Sanfelipe J, Ibarz Servio L, Batalla Cadira JL, Ruiz Marcellán FJ. Characteristics of bladder leiomyoma in our setting. Arch Esp Urol 1995; 48: 987-990.

4. Cornella JL, Larson TR, Lee RA, Magrina JF, KammererDoak D. Leiomyoma of the female urethra and bladder: report of twenty-three patients and review of the literature. Am J Obstet Gynecol 1997; 176: 1278-1285.

5. Park JW, Jeong BC, Seo SI, Jeon SS, Kwon GY, Lee HM. Leiomyoma of the urinary bladder: a series of nine cases and review of the literature. Urology 2010;76:1425-1429.

6. Cornella JL, Larson TR, Lee RA, Magrina JF, KammererDoak D. Leiomyoma of the female urethra and bladder: report of twenty-three patients and review of the literature. Am J Obstet Gynecol 1997; 176: 1278-1285.

7. Sherer DM, Gorelick C, Gabbur N, et al. Color Doppler imaging and 3-dimensional sonographic findings of urinary bladder leiomyoma. J Ultrasound Med 2007; 26:667-670.

8. Yang JM, Huang WC. Sonographic findings of acute urinary retention secondary to an impacted pelvic mass. J Ultrasound Med 2002; 21: 1165-1169.

9. Aytaç SK, Ozcan H. Effect of color Doppler system on the twinkling sign associated with urinary tract calculi. J Clin Ultrasound 1999; 27: 433-439.

10. Chen M, Lipson SA, Hricak H. MR imaging of benign mesenchymal tumours of the urinary bladder. AJR Am J Roentgenol 1997; 168: 399-403.

11. Fasih N, Prasad Shanbhogue AK, Macdonald DB, et al. Leiomyomas beyond the uterus: unusual locations, rare manifestations. Radiographics 2008; 28: 1931-1948.

12. Patel NS, Blick C, Kumar PV, Malone PR. The diagnostic value of abdominal ultrasound, urine cytology and prostatespecific antigen testing in the lower urinary tract symptoms clinic. Int J Clin Pract 2009; 63: 1734-1738.

13. Stamatiou K, Papadoliopoulos I, Dahanis S, Zafiropoulos G, Polizois K. The accuracy of ultrasonography in the diagnosis of superficial bladder tumors in patients presenting with hematuria. Ann Saudi Med 2009; 29: 134-137.

14. Horstman WG, McFarland RM, Gorman JD. Color Doppler sonographic findings in patients with transitional cell carcinoma of the bladder and renal pelvis. J Ultrasound Med 1995; 14: 129-133. 